\title{
Fabrication and Characterization of Photonic Devices Directly Written in Glass Using Femtosecond Laser Pulses
}

\author{
Catalin Florea, Member, IEEE, Member, OSA, and Kim A. Winick, Senior Member, IEEE, Member, OSA
}

\begin{abstract}
Both straight and curved waveguides are written in a variety of silicate glasses using near-IR femtosecond laser pulses. Writing parameters are identified that produce waveguides that support only a single mode and yield smooth-mode profiles. The laser pulse-induced refractive index change is reconstructed from near-field mode profile data using the scalar wave equation and by refractive near-field profiling. Waveguide propagation losses are determined by throughput and Fabry-Pérot resonator measurements. Both coarse and fine period gratings are written and characterized, and the thermal stability of these gratings is investigated. The utility of the femtosecond writing technique is demonstrated by fabricating an optical interleaver.
\end{abstract}

Index Terms-Femtosecond direct writing, laser processing, micromachining, planar optical waveguides, ultrafast laser processing, waveguide fabrication.

\section{INTRODUCTION}

$\mathbf{T}$ HE USE OF femtosecond laser pulses to directly induce refractive index changes in transparent dielectric materials is a new and attractive technique to fabricate active and passive integrated optics devices in glass. Passive optical waveguides [1], [2], $Y$-junction splitters [3], $X$-couplers [4], long-period gratings [5], holograpic gratings [6], birefringent structures [7], rare earth-doped waveguide amplifiers [8], and an optical interleaver [9] have all been recently demonstrated. The use of femtosecond laser-pulse writing is of great interest since this technique, unlike the continuous-wave (CW) or quasi-CW UV exposure methods that are widely used for fibers, is not limited to UV photosensitive glasses. Waveguides have been written in a variety of glass types, including low phonon hosts such as fluorides and highly nonlinear glasses such as chalcogenides [2]. In addition, by appropriately choosing the wavelength of the femtosecond laser, large penetration depths can be achieved. This permits the formation of truly three-dimensional structures [4], [10], [11] rather than simple near-surface planar devices.

Although laser-induced breakdown and damage in transparent materials are well researched subjects, the mechanism that leads to the refractive index changes is not completely

Manuscript received April 25, 2002; revised August 15, 2002. This work was supported by the Department of the Air Force under Contract F29601-99-C-0044 and the National Science Foundation through Grant ECS-9522200.

C. Florea was with the Applied Physics Program, University of Michigan, Ann Arbor, MI 48104 USA. He is now with the IMRA America, Inc., Ann Arbor, MI 48105 USA (e-mail: cflorea@imra.com).

K. A. Winick is with the Department of Electrical and Computer Engineering, University of Michigan, Ann Arbor, MI 48104 USA (e-mail: winick@eecs.umich.edu).

Digital Object Identifier 10.1109/JLT.2003.808678 understood [12]-[15]. Furthermore, the mechanism may depend on the repetition rate employed for the writing process. From a device perspective, this technology is still in its early stages. In particular, fabricated waveguides have yet to be fully characterized either in terms of their propagation losses or the induced refractive-index structures that are responsible for waveguide formation. Waveguide loss is one of the most important parameters in practice, and the loss at a wavelength of $1.55 \mu \mathrm{m}$ is of particular interest for photonics devices that support the optical telecommunications market.

In this paper, we present refractive index profiles of directly written waveguides determined indirectly from measured mode field data and directly from refractive near-field profiling. Propagation loss data are also determined using both throughput and waveguide Fabry-Pérot resonator measurements. We report directly written gratings with periods as short as $2 \mu \mathrm{m}$, and we study the thermal stability of $10 \mu \mathrm{m}$ period gratings. Finally, a fully functional optical interleaver, directly written in fused silica, is described and characterized.

\section{Device FAbrication}

Several glasses were investigated in this work, including fused silica, sodium-alumino-borosilicate, Nd-doped sodiumalumino-borosilicate, BK7, and a commercial Nd-doped silicate laser rod. Most of the devices reported in this paper were written using a Clark-MXR laser system that delivers $150 \mathrm{fs}$ pulses at $775 \mathrm{~nm}$ with a $1-\mathrm{KHz}$ repetition rate. We chose, however, to run the system at a $250-\mathrm{Hz}$ repetition rate for the work reported here. Pulse energies in the range of few $\mu \mathrm{J}$ 's were used for writing, although energies up to the $1 \mathrm{~mJ}$ range were available from the laser. The waveguide shown in Fig. 8 was written using a Ti:sapphire laser system, generating 120 -fs pulses at $790 \mathrm{~nm}$, with a repetition rate of $238 \mathrm{KHz}$. The gratings and the waveguide shown in Fig. 8 were written with the laser beam incident upon the top surface of the substrate. The spot was focused inside the substrate using a lens, and the writing was done by translating the substrate in a direction orthogonal to that of the propagating beam. Several straight waveguides were also written with the laser beam incident upon the end of the substrate. In this configuration, the spot was focused inside the sample using a lens and the writing was done by translating the lens along the direction of the propagating beam. Both of these writing geometries, referred to as transverse writing (TW) and longitudinal writing (LW), respectively, are illustrated in Fig. 1(a) and (b). Under some situations, the focused spot was repeatedly scanned along the 


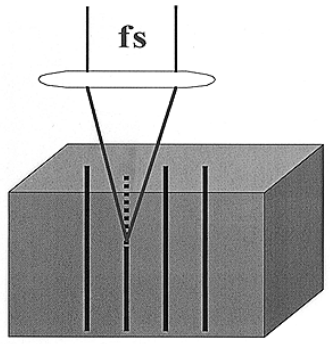

(b)

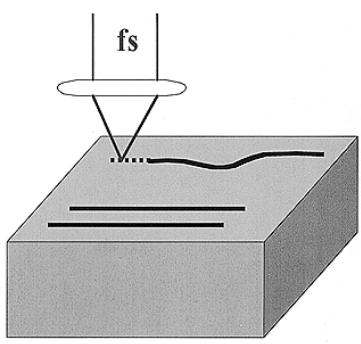

Fig. 1. Writing geometries: (a) Longitudinal and (b) transversal.

same path [8]. The waveguides were written to within several hundred microns of the end faces of the substrate when using the LW geometry in order to avoid dielectric breakdown at the substrate-air interface. The ends of the substrate were then polished down to meet the waveguides. From a practical perspective, it is desirable to use a high repetition rate laser system and the TW geometry, since the former permits rapid device writing, while the latter makes it easier to write curved structures. Circularly symmetrical waveguides, however, are more easily obtained using the LW geometry. We have found it useful to characterize the writing parameters according to the following four criteria:

- writing geometry: transverse (TW) or longitudinal (LW);

pulse repetition rate: low repetition rate (LRR) is several hundred $\mathrm{Hz}$ up to approximately $1 \mathrm{MHz}$ and high repetition rate (HRR) is approximately $1 \mathrm{MHz}$ and above;

- pulse energy: low energy (LE) is nanojoules and high pulse energy (HE) is microjoules;

- scan speed: slow scan (SS) is 5-200 $\mu \mathrm{m} / \mathrm{s}$ and fast scan (FS) is $1-50 \mathrm{~mm} / \mathrm{s}$.

We suspect that cumulative heating due to successive pulses is present in HRR writing regime but absent in LRR.

\section{NEAR-FIELD ModE ANALYSIS}

Near-field mode profiles were obtained by imaging the end of the waveguide onto a CCD array using a microscope objective. The nonlinear response of the CCD array was carefully calibrated at all wavelengths at which mode profiles were measured, and the effective CCD pixel size was also determined. A typical mode profile of a good-quality waveguide written in the LW, LRR, HE, SS regime is shown in Fig. 2. This profile was obtained for a waveguide written in the Nd-doped sodium-alumino-borosilicate glass using a focusing lens with a $25-\mathrm{mm}$ focal length (beam size in front of lens is $7.0 \mathrm{~mm}$ FWHM intensity), $2 \mu \mathrm{J}$ per pulse, a scan speed of $200 \mu \mathrm{m} / \mathrm{s}$ and ten scan repetitions.

By examining near-field mode profiles, we were able to determine conditions that yielded smooth single-mode high-throughput waveguides in the glasses under investigation. The near-field mode profiles can be quite sensitive to the writing parameters, as illustrated in Fig. 3. Note that for this glass the mode takes on a very complex structure at writing pulse energies greater than approximately $4 \mu \mathrm{J}$, and it is very weakly confined at pulse energies below approximately $1 \mu \mathrm{J}$. The mode profiles were also used to investigate the effect of weak

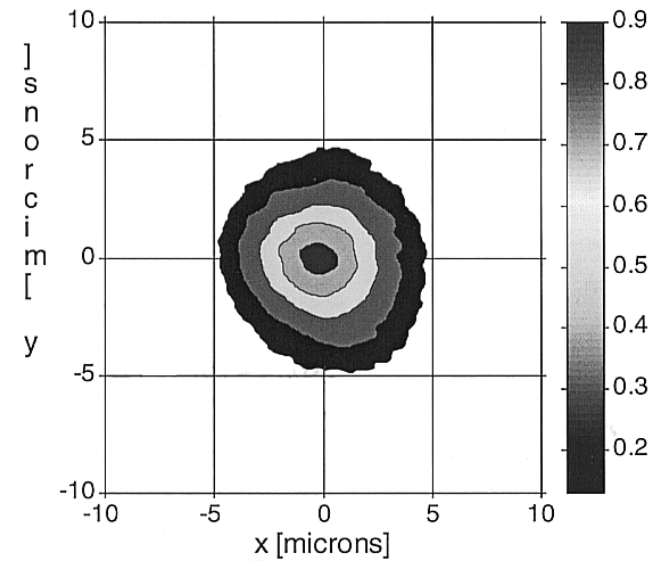

Fig. 2. Single-mode near-field profile at $633 \mathrm{~nm}$ for a good-quality directly written waveguide in $\mathrm{Nd}$-doped sodium-alumino-borosilicate glass $(25-\mathrm{mm}$ focal length lens, $2 \mu \mathrm{J}$ per pulse, ten scans at $200 \mu \mathrm{m} / \mathrm{s}$ ). The shaded bands in concentric rings about the center represent $0.9,0.7,0.5,0.3$, and 0.13 of the peak value, respectively.
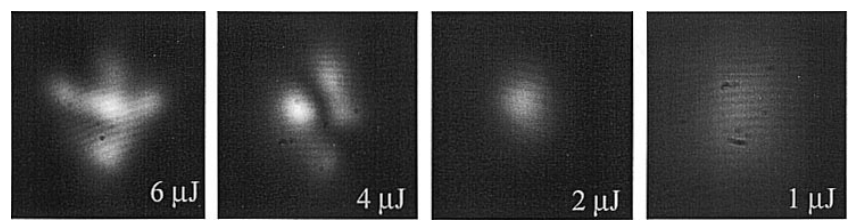

Fig. 3. Mode-profile evolution at $633 \mathrm{~nm}$ as a function of the pulse energy (same writing conditions as in Fig. 2, except for the varying pulse energy).

thermal annealing on the waveguides written in a $\mathrm{Nd}$-doped silicate glass laser rod and in BK7. In the case of the silicate laser rod (3 $\mu \mathrm{J} /$ pulse, $25-\mu \mathrm{m} / \mathrm{s}$ scan speed, five scans, a $25-\mathrm{mm}$ focal length lens with a $e^{-2}$ intensity diameter of $3.6 \mathrm{~mm}$ before the lens), weak thermal annealing at approximately $140{ }^{\circ} \mathrm{C}$ for $50 \mathrm{~min}$ did not alter the shape of the mode profiles but increased the $e^{-2}$ full-width intensity by $15 \%$ to $25 \%$ without improving the waveguide throughput. Weak annealing at $150{ }^{\circ} \mathrm{C}$ for 60 min of waveguides written in BK7 did not improve the throughput nor did it alter the mode profile. This observation is consistent with the fact that it takes more energy per pulse to write waveguides in BK7 than in the $\mathrm{Nd}$-doped silicate laser glass. In the BK7 glass, the mode profile size started to change only after an additional two annealing steps. The first anneal was performed at approximately $200{ }^{\circ} \mathrm{C}$ for 60 min followed by a second 60 min anneal at approximately $260{ }^{\circ} \mathrm{C}$. Some of the more weakly guiding waveguides in the BK7 sample were completely erased after a third anneal at approximately $300{ }^{\circ} \mathrm{C}$ for $60 \mathrm{~min}$. The annealing temperatures discussed previously and elsewhere in this paper were chosen somewhat arbitrarily. We also note that it is difficult to write high-quality waveguides in BK7.

\section{REFRACTIVE INDEX PROFILE RECONSTRUCTION}

Using the measured near-field mode profile data, we have determined the refractive index profile by inverting the scalar-wave equation [16]. The refractive-index profile $n(x, y)$ is given by

$$
n(x, y) \approx N_{\mathrm{eff}}-\frac{\nabla^{2} \sqrt{I(x, y)}}{2 n_{s} k_{o}^{2} \sqrt{I(x, y)}}
$$




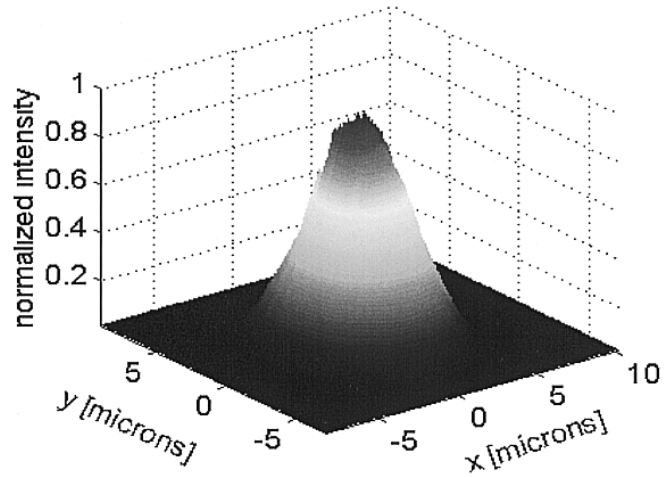

Fig. 4. Single-mode near-field profile at $980 \mathrm{~nm}$ for a Corning SMF-28 fiber.

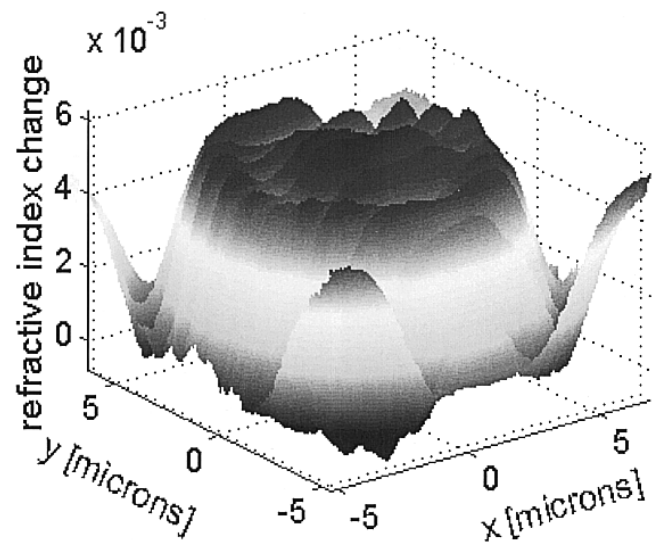

Fig. 5. Reconstructed refractive-index profile for the Corning SMF-28 fiber.

where $k_{0}=2 \pi / \lambda$ is the free-space wavenumber, $n_{s}$ is the substrate refractive index, $N_{\text {eff }}$ is the effective index of the guided mode, and $I(x, y)$ is the mode intensity profile as a function of the transverse $(x, y)$ coordinates. Equation (1) can be evaluated numerically using a finite difference approach.

Due to the second-order derivatives involved in (1), obtaining accurate refractive index profiles requires excellent mode profile data that is very precisely focused on the image collector (a CCD array) and is very low noise. We used extensive averaging in order to obtain good-quality images, and the averaged image was subsequently filtered using a low-pass filter with transfer function $H(\omega)=\sqrt{1 /\left(1+\omega^{4}\right)}$. The inversion procedure was tested on a Corning SMF-28 fiber, which has an $8.3 \mu \mathrm{m}$ core diameter, a step-index profile with an index step $\Delta n$ of 0.0055 and a cladding refractive index of approximately 1.53. The near-field single-mode profile (an average of 25 images) of this fiber is shown in Fig. 4. The mode has a $e^{-2}$ full-width intensity diameter of $\sim 9.5 \mu \mathrm{m}$. This data was collected at a wavelength of $980 \mathrm{~nm}$ by imaging the end of the waveguide onto a CCD array using a $60 \times$ microscope objective. The objective lens was placed approximately one focal length behind the end of the waveguide, and the magnification of this imaging system was computed to be approximately 224 . The fiber was quite short and due care was taken to only excite the fundamental mode. As can be seen in Fig. 5, the recovered index profile $\Delta n(x, y)=n(x, y)-N_{\text {eff }}$ is also step-index, with a $e^{-2}$ intensity diameter of about $9 \mu \mathrm{m}$ and an index step size of about 0.0060 . These values are in very good agreement with the

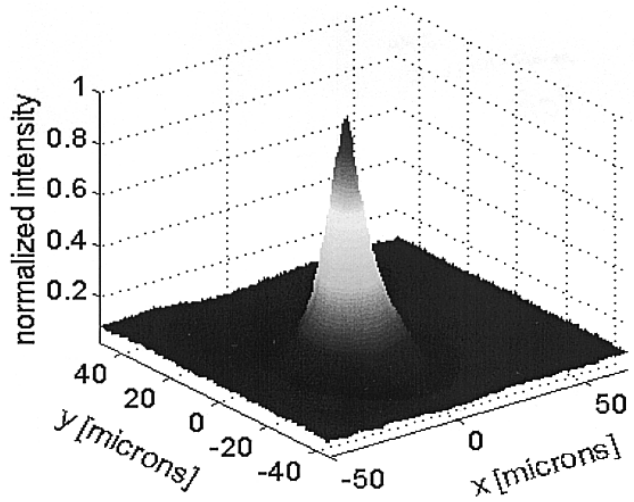

Fig. 6. Single-mode near-field profile at $633 \mathrm{~nm}$ for a directly written waveguide (25-mm focal-length lens, $3 \mu \mathrm{J}$ per pulse, five scans at $25 \mu \mathrm{m} / \mathrm{s}$ ).

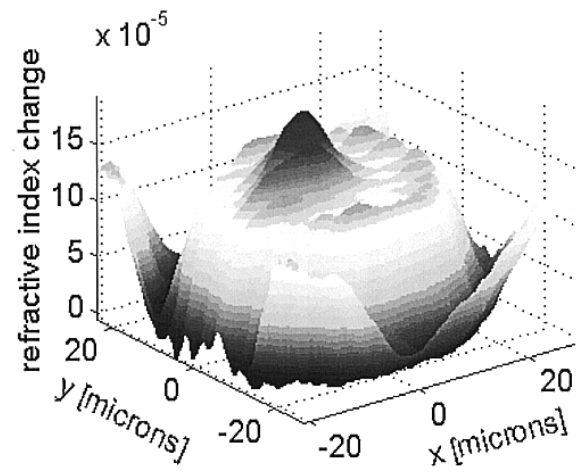

Fig. 7. Change in the refractive index of the directly written waveguide illustrated in Fig. 6.

manufacturer's specifications. We note, however, that we were not able to recover the narrow dip in the center of the refractive index profile which is characteristic of SMF-28 fiber. The "wings" that appear outside of the main step of the recovered profile are an artifact of the low-pass filtering operation. This fact was verified by applying the inversion procedure to the analytic expression for the intensity $I(r)$ of the $L P_{01}$ mode of a weakly guiding, round, step-index optical fiber given by [17]

$$
I(r)=\left\{\begin{array}{c}
{\left[\frac{J_{0}(\kappa r)}{J_{0}(\kappa a)}\right]^{2}, 0 \leq r \leq a} \\
{\left[\frac{K_{0}(\gamma r)}{K_{0}(\gamma a)}\right]^{2}, a \leq r<\infty}
\end{array}\right\}
$$

where

a core radius;

$n_{\mathrm{co}}$ refractive index of the core;

$n_{\mathrm{cl}} \quad$ refractive index of the cladding;

$J_{\nu}()$ Bessel function of the first kind of order $\nu$;

$K_{\nu}()$ modified Bessel function of the second kind of order $\nu$

$\lambda \quad$ vacuum wavelength.

The mode-dispersion relationship is given by

$$
\begin{aligned}
\frac{J_{0}(\kappa a)}{\kappa J_{1}(\kappa a)} & =\frac{K_{0}(\gamma a)}{\gamma K_{1}(\gamma a)} \\
(\kappa a)^{2}+(\gamma a)^{2} & =\left(\frac{2 \pi}{\lambda} a\right)^{2}\left(n_{\mathrm{co}}^{2}-n_{\mathrm{cl}}^{2}\right) .
\end{aligned}
$$




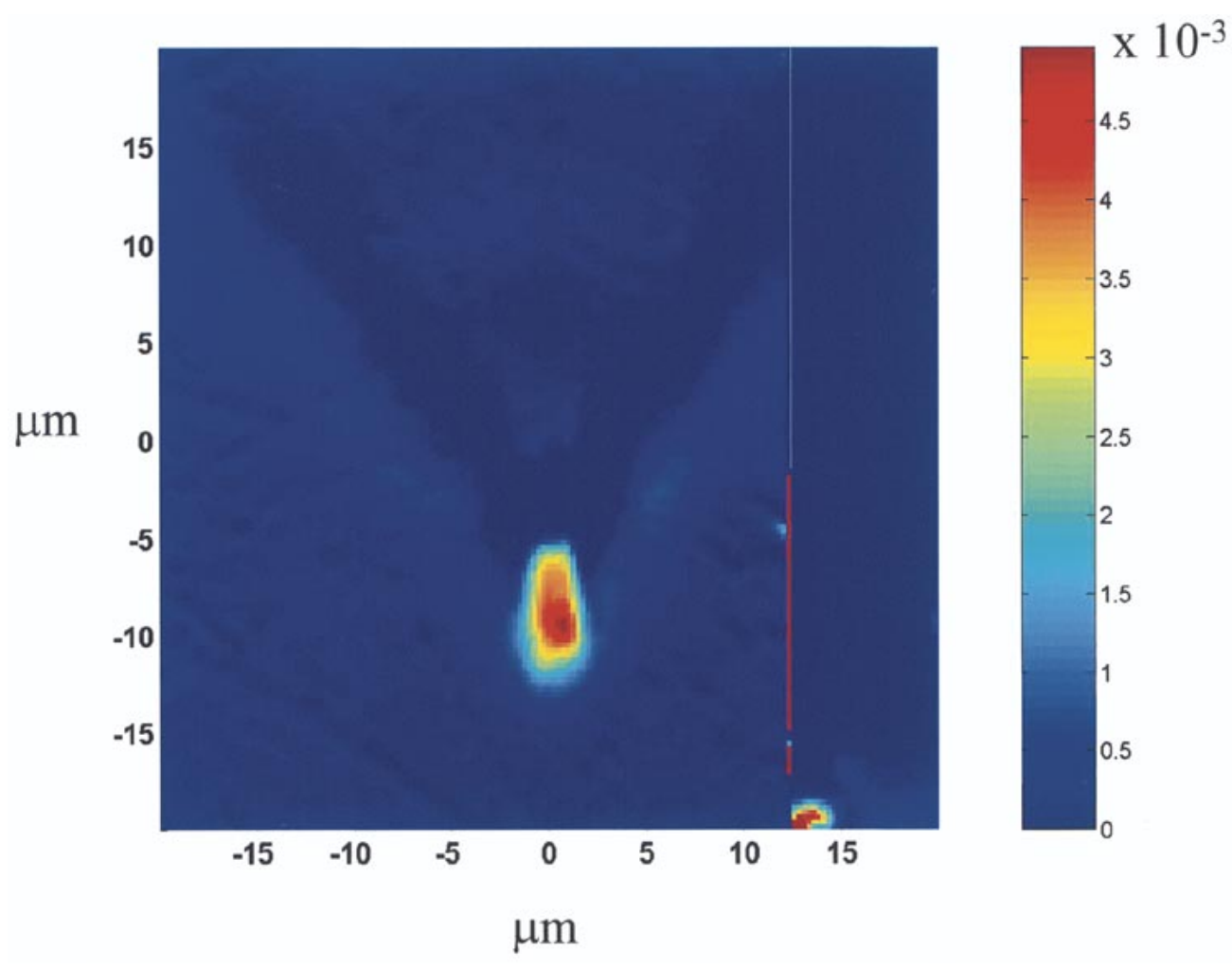

Fig. 8. Refractive-index profile of a directly written waveguide in fused silica (TW, a $0.55 \mathrm{NA}$ lens, $10 \mu \mathrm{J} / \mathrm{pulse}$ at $150 \mathrm{fs}, 238-\mathrm{kHz}$ repetition rate, four scans at $100 \mathrm{~mm} / \mathrm{s}$ ) obtained using a RNF profilometer.

The inversion technique was subsequently applied to one of the Nd-doped silicate glass waveguides mentioned earlier. A plot of a the near-field mode profile at $632.8 \mathrm{~nm}$ is shown in Fig. 6. The waveguide corresponding to Fig. 6 was written with $3-\mu \mathrm{J}$ pulses using the Clark-MXR system described earlier. A focusing lens with a $25-\mathrm{mm}$ focal length (laser beam $e^{-2}$ diameter of $3.6 \mathrm{~mm}$ before the lens) was used, together with a scan speed of $25 \mu \mathrm{m} / \mathrm{s}$ and five scans per waveguide. The writing step was followed by a weak 50 min thermal anneal at approximately $140^{\circ} \mathrm{C}$ as described earlier. The near-field mode profile was imaged onto a CCD array with a $43 \times$ microscope objective and 99 images were averaged. As seen in Fig. 6, the waveguide mode is almost circular and has an $e^{-2}$ full-width intensity diameter of $\sim 32 \mu \mathrm{m}$. The reconstructed refractive-index profile $\Delta n(x, y)=n(x, y)-N_{\text {eff }}$ is nearly step-index with a diameter of approximately $40 \mu \mathrm{m}$, as shown in Fig. 7. A closer inspection of the refractive index change reveals a step-like rise accompanied by an additional peak somewhat off-center. The peak-to-peak index difference is approximately $2 \times 10^{-4}$. Although a $40 \mu \mathrm{m}$ diameter, step-index waveguide with a $\Delta n$ of 0.0002 would have a $V$ number of about 4.8 and hence, should be multimode at $6328 \mathrm{~nm}$, we were unable to excite higher order modes. One possible explanation for this observation is that the mode overlap between the launched pump and the higher order modes may have been poor.

A commercial refractive near-field (RNF) profilometer ${ }^{1}$ was also used to examine some waveguides written in fused silica. The results are shown in Fig. 8. The elliptical shape of the mode is due to the TW geometry used to write the waveguide.

${ }^{1}$ Rinck elektronik, Jena, Germany (http://www.rinck-elektronik.de).

\section{WAVEguide PROPAgation Losses}

We measured propagation losses for directly written waveguides at two different wavelengths, $632.8 \mathrm{~nm}$ and $1560 \mathrm{~nm}$. We did not, however, measure the same devices at both of these two wavelengths, and therefore we do not know the dependence of loss upon the wavelength.

The loss at $632.8 \mathrm{~nm}$ was evaluated by making throughput measurements on three different length straight waveguides all written under the same conditions in three separate but identical substrates. Due care was taken to assure that identical coupling conditions were achieved for each of three waveguides. The three different samples were mounted using the same optical setup and the back-reflection from the input face of each sample was centered on a fixed pinhole, thus assuring the same angular alignment for each sample. The waveguides were written in the same Nd-doped silicate laser glass rod mentioned earlier and with the same writing parameters as the ones for the waveguide illustrated in Fig. 6. These waveguides, however, were not thermally annealed. The throughput data as a function of waveguide length is shown in Fig. 9. The data corresponds to a propagation loss of about $1.35 \mathrm{~dB} / \mathrm{cm}\left(0.31 \mathrm{~cm}^{-1}\right)$ and a coupling efficiency of about $44 \%$. We estimate that our throughput measurements are in error by no more than 5\% and thus, we deduce that the accuracy of our reported loss value is within $20 \%$ of the actual value. Similar measurements made on other sets of waveguides yielded points with considerable scatter about the straight line fit. We have, in fact, seen throughput variations as large as $25 \%$ under the same coupling conditions for waveguides written with the same parameters in the same sample. At the present time, we are unable to explain the origin of these variations. It is possible 


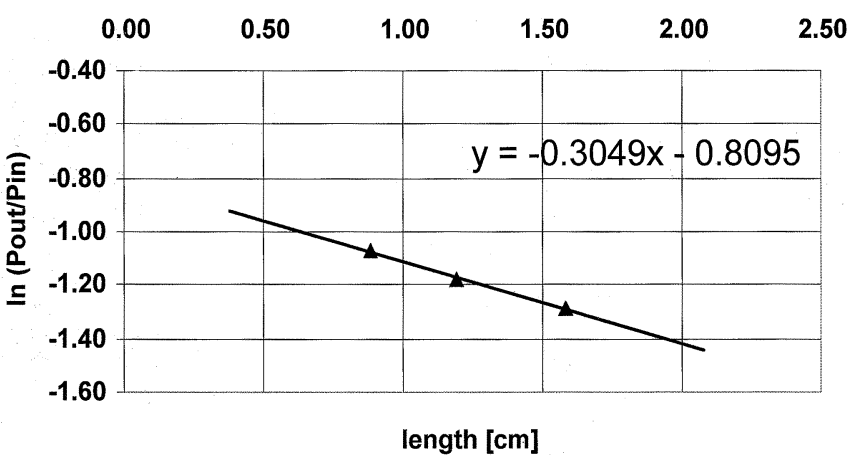

Fig. 9. Throughput data at $633 \mathrm{~nm}$ as a function of waveguide length.

that even a small amount of mechanical wobble of the focusing lens during translation may contribute to the loss.

The loss at $1560 \mathrm{~nm}$ was measured using the simpler and more reliable Fabry-Pérot method [18]. A Fabry-Pérot cavity is constructed out of a single-mode waveguide together with its polished endfaces (or mirrors attached to the endfaces). The fraction $T$ of incident monochromatic light, which is transmitted through this cavity depends on the cavity losses and the cavity roundtrip phase delay $\phi$. More precisely

$$
\begin{aligned}
T & =\gamma \frac{e^{-\alpha L}\left(1-R_{1}\right)\left(1-R_{2}\right)}{\left(1-e^{-\alpha L} \sqrt{R_{1} R_{2}}\right)^{2}+4 \sqrt{R_{1} R_{2}} e^{-\alpha L} \sin ^{2} \phi} \\
\phi & =\frac{2 \pi}{\lambda} 2 L N_{\text {eff }}
\end{aligned}
$$

where $R_{1}, R_{2}$ are the endface reflectivities, $\alpha$ is the propagation loss in $\mathrm{cm}^{-1}, L$ is the length of the cavity in $\mathrm{cm}, \lambda$ is the wavelength of the monochromatic light, $N_{\text {eff }}$ is the effective index of the guided mode, and $\gamma$ is a constant that depends on the efficiency with which the light is launched into the cavity. It follows from (5) that $T$ is a periodic function of $\phi$ with period $\pi$. $T$ achieves its maximum value $T_{\max }$ when $\phi=0$ and its minimum value $T_{\min }$, when $\phi=\pi / 2$. Equation (5) may be inverted to obtain the propagation loss in terms of $T_{\max }$ and $T_{\min }$

$$
\alpha\left[\frac{\mathrm{dB}}{\mathrm{cm}}\right]=-\frac{4.34}{L[\mathrm{~cm}]} \ln \frac{1-\sqrt{1-K^{2}}}{K \sqrt{R_{1} R_{2}}}
$$

where

$$
K=\frac{I_{\max }-I_{\min }}{I_{\max }+I_{\min }}
$$

is the fringe contrast, and $I_{\max }$ and $I_{\min }$ are the maxima and the minima of the transmitted light through the cavity as the phase $\phi$ is varied over an interval of at least $\pi$. A significant advantage of the Fabry-Perot loss measurement technique is that the calculation of the loss using (7) does not require knowledge of the coupling efficiency $\gamma$.

The phase may be varied by either temperature tuning the substrate, which will alter the refractive index of the glass and the waveguide length through expansion or contraction, or by tuning the wavelength of the monochromatic source. We experimented with both techniques but found that it was easier to implement wavelength tuning, since during temperature tuning the coupling conditions may change. As the wavelength is tuned over a range $\Delta \lambda$, the corresponding change $\Delta \phi$ in the roundtrip phase delay is given by

$$
\Delta \phi=-\frac{4 \pi}{\lambda^{2}} L N_{\mathrm{eff}} \Delta \lambda .
$$

The result given by (5) assumes that the endfaces of the cavity are perpendicular to the waveguide. Any deviation from perpendicularity will induce additional losses in the cavity because the mode, upon reflection form an endface, will not be coupled with $100 \%$ efficiency back into the waveguide. Assuming that the waveguide mode has a Gaussian intensity profile of known size this additional loss can be quantified as a function of the angular deviation $\theta$ of the endfaces from perpendicularity [19]

$$
\operatorname{loss}[\mathrm{dB}]=42.8\left(\frac{n \rho}{\lambda} \theta\right)^{2}
$$

where $\lambda$ equals the vacuum wavelength of the mode, $n$ is the nominal refractive index of the waveguide, $\rho$ equals the $1 / e^{2}$ full-width intensity of the gaussian mode, and $\theta$ equals the angular deviation (in radians) of the endface from perpendicularity with the waveguide. Thus, if endface perpendicularity is not perfectly achieved, then (7) gives an upper bound for the propagation loss rather than the propagation loss itself. We have been able to achieve perpendicularity to within approximately $0.2^{\circ}$. Using measured-mode size data together with (10), we estimated that the additional loss due to nonperpendicularity should not contribute more than $0.2 \mathrm{~dB} / \mathrm{cm}$ to the computed value of $\alpha$ for samples of about $1 \mathrm{~cm}$ in length.

Our measurements were performed using a single-frequency, linearly polarized, diode laser (Coherent model 2010M) with a $0.1-\mathrm{MHz}$ linewidth. This laser could be manually wavelength tuned from $1530 \mathrm{~nm}$ to $1570 \mathrm{~nm}$, and a fine tuning range of $30 \mathrm{GHz}$ (e.g., $0.243 \mathrm{~nm}$ at $1560 \mathrm{~nm}$ ) was accessible by means of a PZT-controlled drive mounted on one of the mirrors of the laser. The signal applied to the PZT drive was changed very slowly in order to insure that the movement of the mirror was smooth enough to produce a continuous change in the frequency of the lasing mode. Under these conditions, the roundtrip phase delay varied almost linearly in time resulting in a sinusoidal modulation of the light passing through the cavity as predicted by (5). A $1560-\mathrm{nm}$ isolator was needed to prevent any feedback into the single-frequency diode laser and a polarizer was used at the output of the waveguide in order to select only TM polarized light. All the measurements were done for TM polarization (i.e., polarized perpendicular to the top surface of the substrate). The detected signal from output of the waveguide was normalized with respect to the input signal from the laser source so that any input-power variations, due to the wavelength tuning, could be accounted for.

The Fabry-Pérot measurement technique was first tested and refined on ion-exchanged waveguides in glass. The waveguides were created by $\mathrm{Ag}^{+}$-exchange in a commercially available glass and were cut and polished to a length of $2.06 \mathrm{~cm}$. The waveguides were single-mode at $1560 \mathrm{~nm}$. A typical trace of the light transmitted through the waveguide during wavelength tuning is shown in Fig. 10. For the tuning range of $0.14 \mathrm{~nm}$, the phase-shift at $1560 \mathrm{~nm}$, as given by (9), will be $\Delta \varphi=2 \pi * 3.6$, which means the output power of the waveguide will go through about 3.6 intensity fringes. This result is in agreement with the 


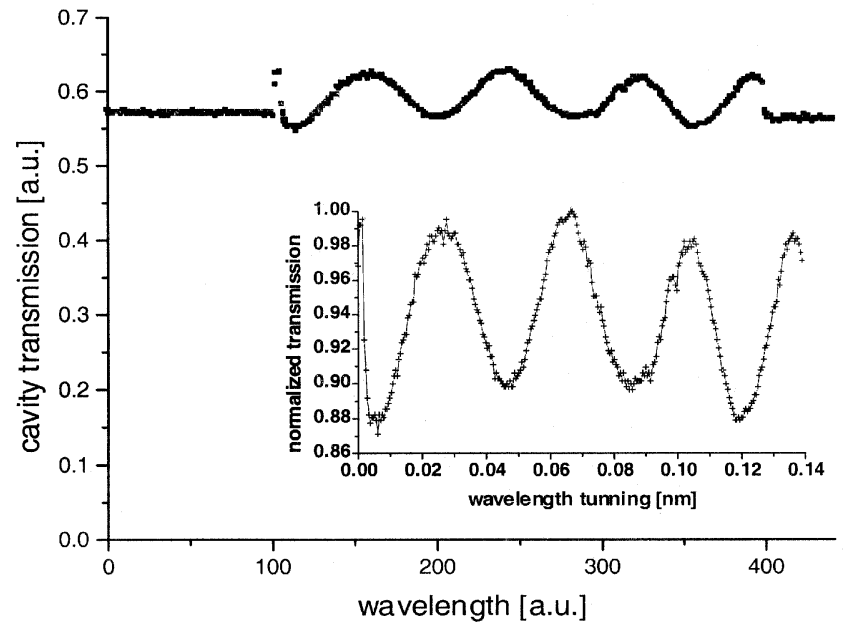

Fig. 10. Fabry-Pérot loss measurement for an $\mathrm{Ag}^{+}$-exchanged glass waveguide at $1560 \mathrm{~nm}$.

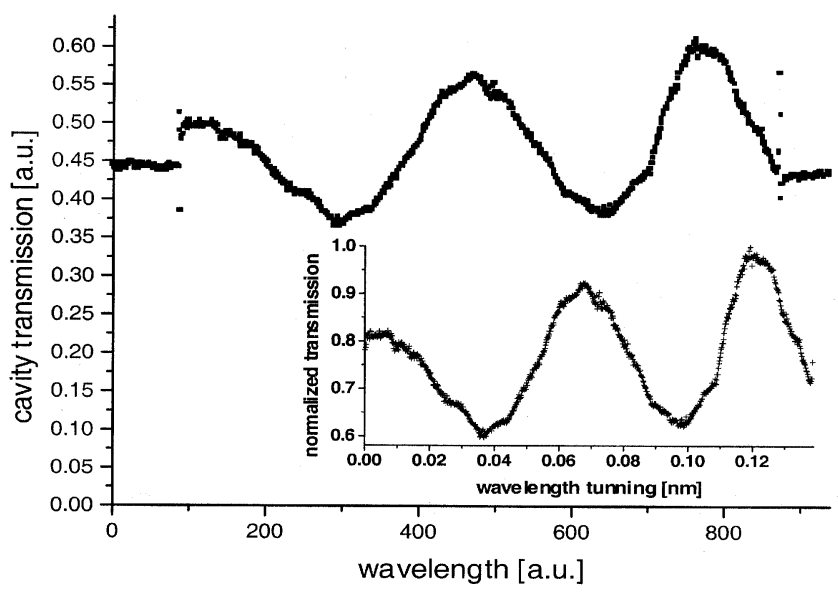

Fig. 11. Fabry-Pérot loss measurement for a directly written waveguide in the $\mathrm{Nd}$-doped alkali-alumino-borosilicate glass at $1560 \mathrm{~nm}$.

measured data shown in Fig. 10. The device was used with bare endfaces and therefore, $R_{1}=R_{2}=0.04$. The measured fringe contrast $K$, was $0.0626(6.3 \%)$, yielding an upper bound on the propagation loss of about $0.51 \mathrm{~dB} / \mathrm{cm}\left(0.12 \mathrm{~cm}^{-1}\right)$, as given by (7). This measured loss is typical of previously reported loss values for potassium ion exchanged waveguides.

In a similar fashion, the propagation loss of directly written waveguides in the $\mathrm{Nd}$-doped sodium-alumino-borosilicate glass were measured. The data shown in Fig. 11 is for a $1.2-\mathrm{cm}$-long waveguide written with $6-\mu \mathrm{J}$ pulse energies, a $25-\mathrm{mm}$ focal-length lens (beam size is $3.5 \mathrm{~mm}$ FWHM intensity), at a $200-\mu \mathrm{m} / \mathrm{s}$ scan speed and ten scans. In order to improve the fringe contrast, a $95 \%$ reflector was attached to the output endface of the waveguide. The measured fringe contrast $K$ was $21.7 \%$, yielding a propagation loss value (an upper bound) of $2.1 \mathrm{~dB} / \mathrm{cm}\left(0.49 \mathrm{~cm}^{-1}\right)$. It was estimated that no more than $0.1 \mathrm{~dB} / \mathrm{cm}$ of this loss could be attributed to the endfaces being nonperpendicular to the waveguide. Since the sample was rather short, some light from the source, not coupled into the waveguide, reached the detector. This background light added a fixed bias term to the denominator of (8). Based on the collected data, this bias could account for

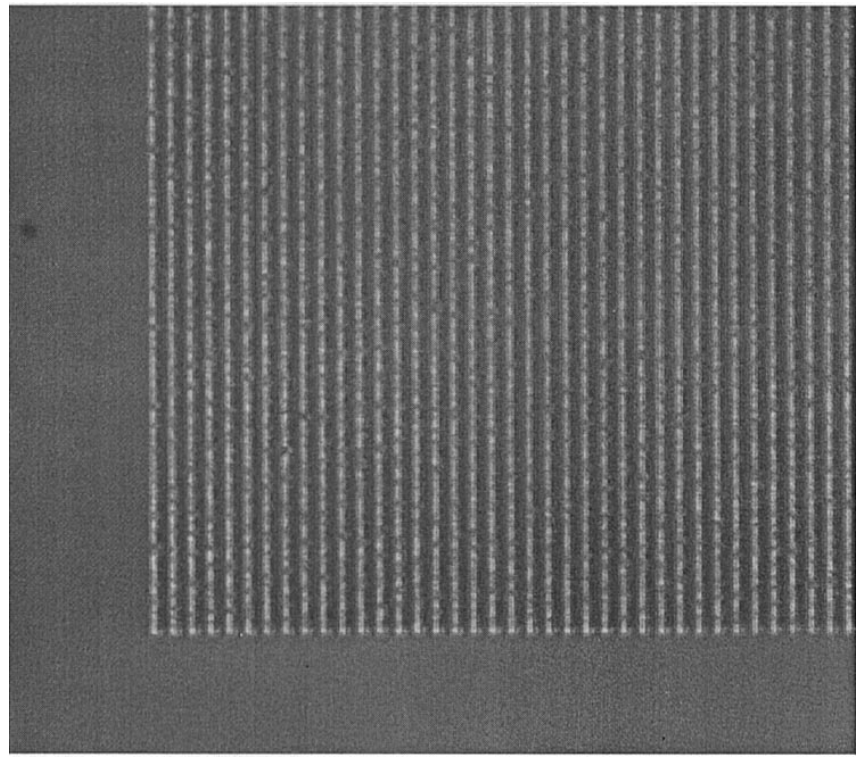

Fig. 12. Phase-contrast micrsocpe image of a $10-\mu \mathrm{m}$ period grating directly written in fused silica with $5 \mu \mathrm{J}$ pulse energy.

up to $0.15 \mathrm{~dB} / \mathrm{cm}$ of the calculated $2.1-\mathrm{dB} / \mathrm{cm}$ propagation loss value reported here.

\section{GRATING FABRICATION AND CHARACTERIZATION}

In addition to waveguides, we have also fabricated gratings using near-IR femtosecond laser pulses. The beam was focused inside the sample and each grating line was drawn by scanning the focused laser beam through the sample in a direction perpendicular to the direction of propagation of the laser beam but parallel to the surface of the sample. The depth of each grating line is determined by the Rayleigh range of the focused laser beam and the threshold intensity of the writing process. The scan rate was $25 \mu \mathrm{m} / \mathrm{s}$ and only a single scan was performed for each grating line. Gratings with a $10-\mu \mathrm{m}$ period were written in BK7 flats with pulse energies of either $2.5 \mu \mathrm{J}$ or $5 \mu \mathrm{J}$ and in fused silica with pulse energies of either $0.5 \mu \mathrm{J}, 1.0 \mu \mathrm{J}, 1.5 \mu \mathrm{J}$, $2.5 \mu \mathrm{J}$, or $5 \mu \mathrm{J}$. A $15-\mathrm{mm}$ focal-length focusing lens was used for $\mathrm{BK} 7$, and a $50 \times$ microscope objective was used for fused silica. The $e^{-2}$ intensity diameter of the beam in front of the focusing lens/microscope objective was approximately $7 \mathrm{~mm}$. A phase-contrast microscope image of a $10-\mu \mathrm{m}$ period grating written into fused silica is shown in Fig. 12. Also, a 2- $\mu \mathrm{m}$ period grating was written in fused silica using a pulse energy of $1 \mu \mathrm{J}$ and a $20 \times$ microscope objective as the focusing lens.

We evaluated the quality of the written gratings by measuring their diffraction efficiencies into the first order using unpolarized normally incident light at a wavelength of $632.8 \mathrm{~nm}$. Diffraction efficiencies (i.e., sum of the power in +1 and -1 orders divided by the incident power) of up to $24 \%$ were observed in BK7 in the case of the $10-\mu \mathrm{m}$ period gratings. The $2-\mu \mathrm{m}$ period grating in fused silica had a diffraction efficiency of only $0.02 \%$. Similar structures with a period of $1 \mu \mathrm{m}$ have been reported elsewhere [7], but they were viewed as induced microlayers and no diffraction-efficiency data was reported.

In fused silica, the relationship between diffraction efficiency and writing-pulse energy was studied and the results are shown 


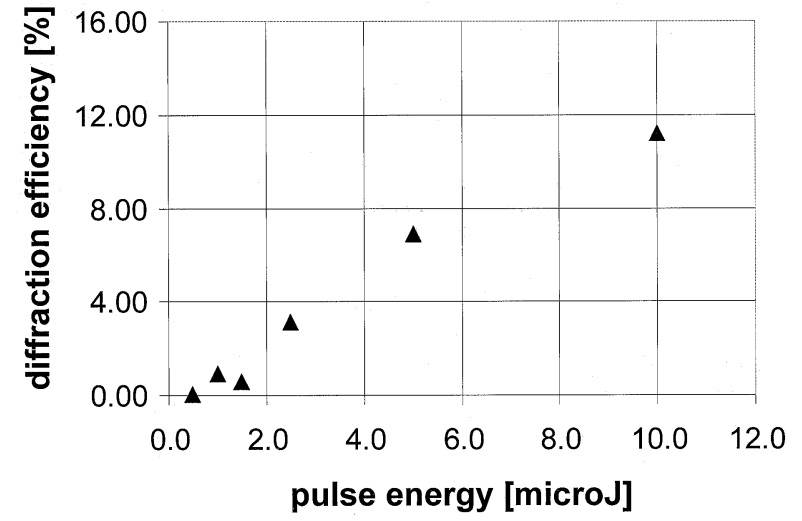

Fig. 13. Diffraction efficiency versus writing-pulse energy for gratings in fused silica.

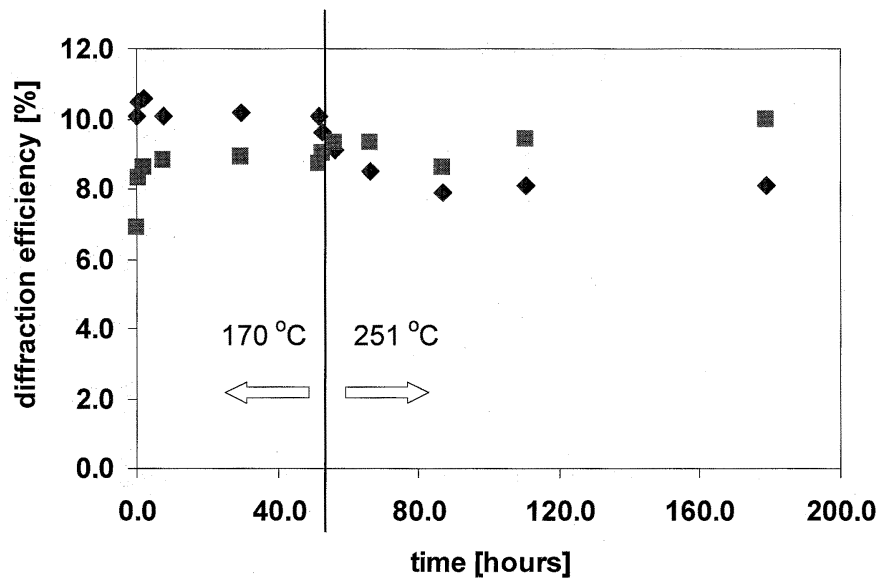

Fig. 14. Thermal annealing of the gratings in fused silica ( $)$ and BK7 $(\bullet)$ Both gratings have $10-\mu \mathrm{m}$ period and were written with 5- $\mu \mathrm{J}$ energy/pulse.

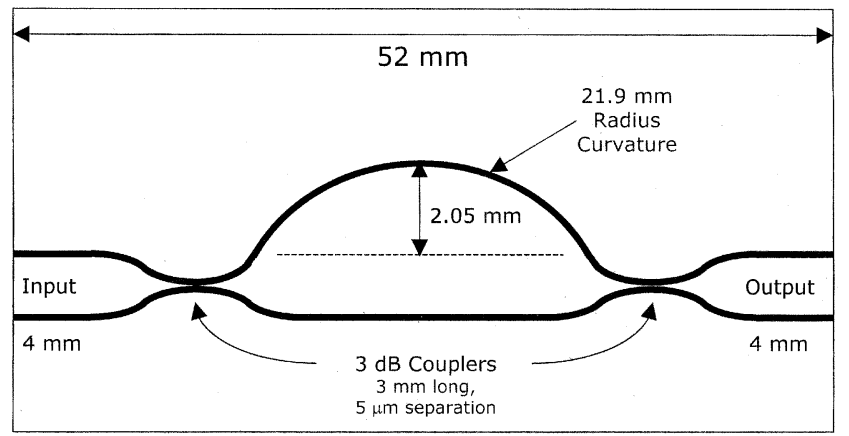

Fig. 15. Schematic of directly written optical interleaver.

in Fig. 13 for the $10-\mu \mathrm{m}$ period gratings. The fused silica gratings have shown a pronounced polarization dependence, with light polarized parallel to the grating lines being diffracted about four times more strongly than the light polarized perpendicular to the grating lines. This observation is in agreement with earlier reports [7] of highly birefringent structures. However, we did not see this effect in the BK7 glass.

Thermal annealing studies were performed on the $10-\mu \mathrm{m}$ period gratings in both fused silica and BK7. The gratings were maintained at $170{ }^{\circ} \mathrm{C}$ for approximately $50 \mathrm{~h}$ and at $250{ }^{\circ} \mathrm{C}$ for an additional $130 \mathrm{~h}$. As illustrated in Fig. 14, the diffraction efficiency for the grating fabricated in BK7 exhibits a slight

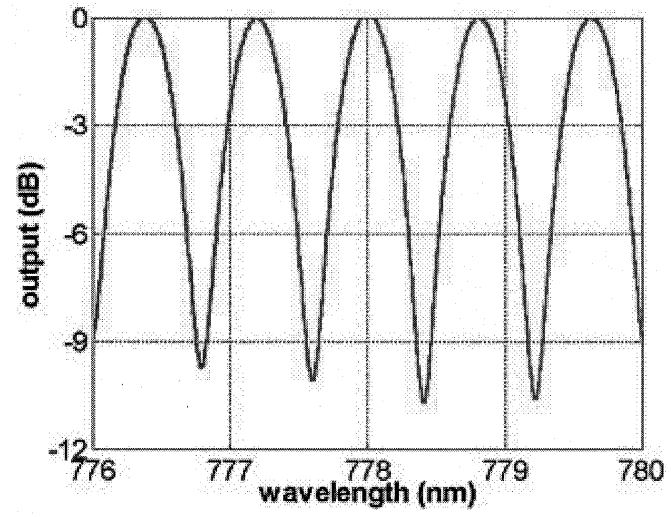

Fig. 16. Single-channel output spectrum from the directly written optical interleaver.

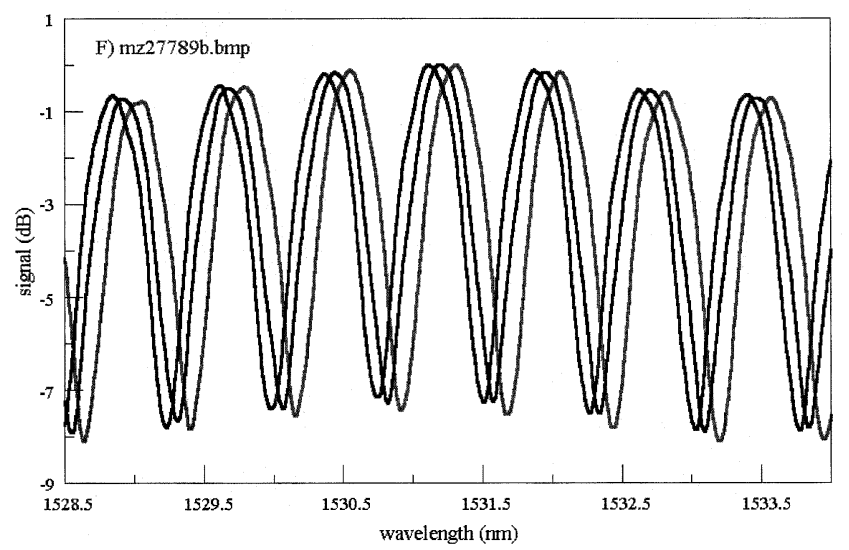

Fig. 17. Interleaver channel tunning. Progressive phase trimming (shifting from left to right on the image) can recenter the interferometric device on the ITU grid or any other performance target.

dip when the anneal temperature is raised to $250{ }^{\circ} \mathrm{C}$, while the diffraction efficiency of the grating made in fused silica remains relatively constant.

\section{OPTICAL INTERLEAVER}

In order to illustrate the capabilites of the direct-write technology, a fully functional integrated optic interleaver was written in fused silica [9], using a LRR laser system. ${ }^{2}$ A schematic diagram of the device is shown in Fig. 15. It consists of an unequal path-length Mach-Zehnder interferometer constructed using two 3-dB couplers. The path-length difference between the two arms is approximately $0.6 \mathrm{~mm}$, which corresponds to a channel spacing of nominally $0.7 \mathrm{~nm}$ at a wavelength of $778 \mathrm{~nm}$. The writing was done in the LRR regime. The interleaver was characterized using a broadband source centered around $776 \mathrm{~nm}$. The output spectrum from a single arm of this device is shown in Fig. 16. The channel spacing can be observed to be approximately $0.75 \mathrm{~nm}$. Femtosecond laser pulses can also be used to "trim" the optical path length of integrated optical devices such as interleavers, which operate on interferometric principles. We were able to tune the channel positions of a commercially produced optical

\footnotetext{
${ }^{2}$ The interleaver was provided by Translume, Inc., Ann Arbor, MI 48108 USA (http:www.translume.com).
} 
interleaver by trimming one of the arms of the Mach-Zehnder interferometer with femtosecond laser pulses while monitoring, in realtime, the spectrum of the device output [9]. Fig. 17 illustrates how the device's spectral response was altered by this trimming process.

\section{CONCLUSION}

Straight and curved waveguides have been written in BK7, fused silica, sodium-alumino-borosilicate, Nd-doped sodium-alumino-borosilicate, and a Nd-doped silicate laser glass. Writing parameters were identified that produced smooth single-mode high-throughput waveguides. The induced refractive-index profiles were recovered from near-field mode profiles by inverting the scalar-wave equation and by using refractive near-field profiling. Propagation losses were measured using both throughput measurements and a Fabry-Pérot resonator technique. The losses were found to be in the range 1.3 to $2.5 \mathrm{~dB} / \mathrm{cm}$ for directly written straight waveguides. The sodium-alumino-borosilicate glass samples that we had for this work were of poor quality. Bulk striations were easily seen by the naked eye in the glass. Although great care was taken to avoid the obviously bad regions, one can not be sure of the actual quality of the medium in which the waveguides were written. We are optimistic that with the proper glass under the proper writing conditions, these losses can be reduced. Gratings with $10-\mu \mathrm{m}$ and $2-\mu \mathrm{m}$ periods were also fabricated and shown to be thermally stable. Finally, an optical interleaver was demonstrated, which illustrates that the direct-write method can be used to fabricate complex integrated optical devices.

\section{REFERENCES}

[1] K. Miura, J. Qiu, H. Inouye, T. Mitsuyu, and K. Hirao, "Photowritten optical waveguides in various glasses with ultrashort pulse laser," Appl. Phys. Lett., vol. 71, pp. 3329-3331, Dec. 1997.

[2] K. Hirao and K. Miura, "Writing waveguides and gratings in silica and related materials by a femtosecond laser," J. Non-Cryst. Solids, vol. 239 , pp. 91-95, Oct. 1998.

[3] D. Homoelle, S. Wielandy, A. Gaeta, N. F. Borrelli, and C. Smith, "Infrared photosensitivity in silica glasses exposed to femtosecond laser pulses," Opt. Lett., vol. 24, pp. 1311-1311, Sep. 1999.

[4] K. Minoshima, A. M. Kowalevicz, I. Hartl, E. P. Ippen, and J. G. Fujimoto, "Photonic device fabrication in glass by use of nonlinear materials processing with a femtosecond laser oscillator," Opt. Lett., vol. 26, pp. 1516-1518, Oct. 2001.

[5] Y. Kondo, K. Nouchi, T. Mitsuyu, M. Watanabe, P. G. Kazansky, and K Hirao, "Fabrication of long-period fiber gratings by focused irradiation of infrared femtosecond laser pules," Opt. Lett., vol. 24, pp. 646-648, May 1999.

[6] K. Kawamura, N. Sarukura, and M. Hirano, "Holographic encoding of fine-pitched micrograting structures in amorphous $\mathrm{SiO}_{2}$ thin films on silicon by a single femtosecond laser pulse," Appl. Phys. Lett., vol. 78, pp. 1038-1040, Feb. 2001

[7] L. Sudrie, M. Franco, B. Prade, and A. Mysyrowicz, "Writing of permanent birefringent microlayers in bulk fused silica with femtosecond laser pulses," Opt. Commun., vol. 171, pp. 279-284, Dec. 1999.

[8] Y. Sikorski, A. A. Said, P. Bado, R. Maynard, C. Florea, and K. Winick, "Optical waveguide amplifier in Nd-doped glass written with near-IR femtosecond laser pulses," Electron. Lett., vol. 36, pp. 226-227, Feb. 2000.
[9] K. Winick, C. Florea, A. A. Said, T. Sosnowski, and P. Bado, "Fabrication of photonic devices in glass using femtosecond pulses," in OSA Annual Meeting, Long Beach, California, Oct. 14-18, 2001, paper ThAA1.

[10] S. Nolte, M. Will, B. N. Chickhov, and A. Tuennermann, "Waveguides produced by ultrashort laser pulses inside glasses and crystals," in Proc. SPIE Photonics West, San Jose, CA, Jan. 20-25, 2002.

[11] P. Bado, "Ultrafast pulses create waveguides and microchannels," Laser Focus World, pp. 73-78, Apr. 2000.

[12] C. B. Schaffer, A. Brodeur, and E. Mazur, "Laser-induced breakdown and damage in bulk transparent materials induced by tightly focused femtosecond laser pulses," Meas. Sci. Technol., vol. 12, pp. 1784-1794, Nov. 2001.

[13] J. W. Chan, T. R. Huser, S. H. Risbud, and D. M. Krol, "Waveguide fabrication in fused silica using tightly focused femtosecond laser pulses," Proc. SPIE, vol. 4640, pp. 129-136, 2002.

[14] J. W. Chan, T. Huser, S. Risbud, and D. M. Krol, "Structural changes in fused silica after exposure to focused femtosecond laser pulses," Opt Lett., vol. 26, pp. 1726-1728, Nov. 2001.

[15] C.-H. Fan and J. P. Longtin, "Modeling optical breakdown in dielectrics during ultrasfast laser processing," Appl. Opt., vol. 40, pp. 3124-3131, Jun. 2001

[16] M. L. von Bibra and A. Roberts, "Refractive index reconstruction of graded-index buried channel waveguides from their mode intensities," J. Lightwave Technol., vol. 15, pp. 1695-1699, Sep. 1997.

[17] A. Snyder and J. Love, Optical Waveguide Theory. New York: Chapman and Hall, 1983, pp. 311-312.

[18] R. Regener and W. Sohler, "Loss in low-finesse Ti: $\mathrm{LiNbO}_{3}$ optical waveguide resonators," Appl. Phys. B. Photophys. Laser Chem., vol. B36, pp. 143-147, Mar. 1985.

[19] A. Snyder and J. Love, Optical Waveguide Theory. New York: Chapman and Hall, 1983, pp. 420-430.

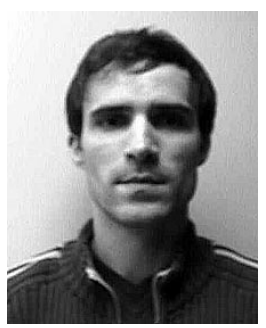

Catalin Florea (S'97-M'02) was born in Bucharest, Romania, in 1972. He received the B.S. degree in physics from Bucharest University, Bucharest, in 1995 and the M.S. degree in electrical engineering and computer science and the Ph.D. degree in applied physics from the University of Michigan, Ann Arbor, in 1999 and 2002, respectively.

From 1996 to 2002, he was a Member of the Integrated Optics Group, University of Michigan, and in 2002, he joined Northstar Photonics, where was involved with glass-integrated optics. Currently, he is employed with IMRA America, Inc., Ann Arbor, MI, where he works on femtosecond fiber laser development. His research interests include planar waveguide devices in rare earth-doped glass and lithium niobate, ion-exchange techniques, laser micromachining, and periodically poled lithium niobate.

Dr. Florea is a Member of the Optical Society of America (OSA).

Kim A. Winick (' '77-M'80-SM'98) was born in New York, NY, on July 27, 1954. He received the B.S. degree in electrical engineering from the Pennsylvania State University, State College, PA, in 1976, and the M.S. and Ph.D. degrees in electrical engineering from the University of Michigan, Ann Arbor, in 1977 and 1981, respectively.

From 1981 to 1988, he was a Member of the Technical Staff of the Lincoln Laboratory, Massachusetts Institute of Technology, Lexington, working on millimeter-wave and optical communication systems. In 1988, he joined the Faculty of the Department of Electrical Engineering and Computer Science, University of Michigan, where he is currently an Associate Professor. His research interests are in the areas of glass and crystal-integrated optics, ultrafast laser micromachining, information theory, and communications.

Dr. Winick is a Member of the Optical Society of America (OSA). 